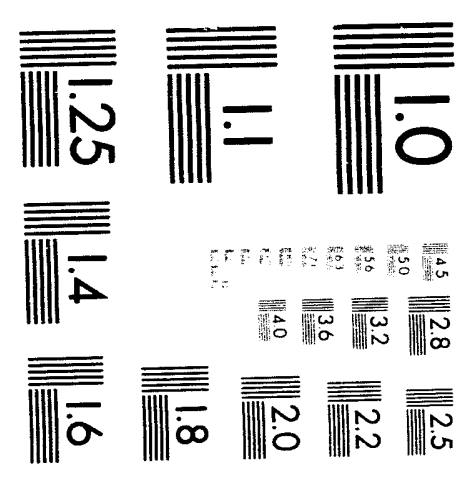



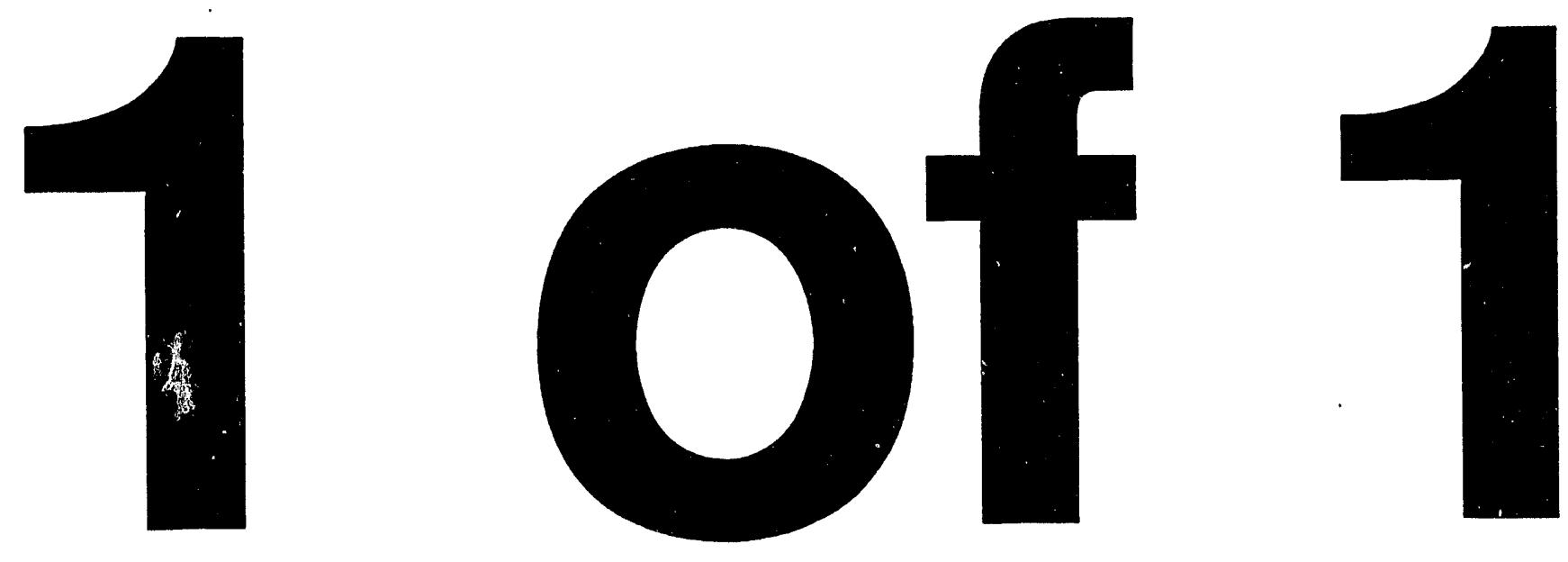


\section{INSTITUTE FOR FUSION STUDIES}

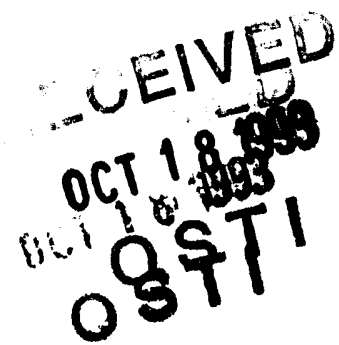

DOE/ET-53088-614

IFSR \#614

An Explanation for Experimental Observations of Harmonic Cyclotron Emission Induced by Fast Ions

K.R. Chen, W. Horton, and J.W. Van Dam Institute for Fusion Studies

The University of Texas at Austin

Austin, Texas 78712

September 1993

\section{THE UNIVERSITY OF TEXAS}

\section{AUSTIN}




\title{
An Explanation for Experimental Observations of Harmonic Cyclotron Emission Induced by Fast Ions
}

\author{
K.R. Chen, W. Horton, and J.W. Van Dam \\ Institute for Fusion Studies \\ The University of Texas at Austin \\ Austin, Texas 78712
}

\begin{abstract}
An explanation, supported by numerical simulations and analytical theory, is given for the harmonic cyclotron emission induced by fast ions in tokamak plasmas - in particular, for the emission observed at low harmonics in deuterium-denterium and deuterium-tritium experiments in the Joint European Tokamak. We show that the first proton harmonic is one of the highest spectral peaks whereas the first alpha harmonic is weak. We also compare the relative spectral amplitudes of different harmonics. Our results are consistent with the experimental observations. The simulations verify that the instabilities are caused by a weak relativistic mass effect. Simulation aleo show that a nonuniform magnetic field leads to no appreciable change in the growth rate and saturation amplitude of the waves.
\end{abstract}

PACS numbers: $52.35 . \mathrm{Qz}, 52.65 .+\mathrm{z}, 52.60 .+\mathrm{h}, 52.55 .-\mathrm{s}$ 
Superthermal radiation by fast ions at harmonics of the local cyclotron frequencies has been observed from tokamak plasmas. ${ }^{1-5}$ The first proton harmonic for the deuterivindeuterium (DD) reaction has one of the strongest-intensity narrow peaks in the power spectrum and is measured to be linearly proportional to the fusion reaction rate over several orders of magnitude. ${ }^{2,3}$ In contrast, the first alpha-particle harmonic in a deuterium-tritium (DT) plasma is weak, and the second alpha-particle (or first proton) harmonic has the highest peak. ${ }^{5,6}$ Because of the importance of the fast ion behavior in a burning fusion reactor, there have been several recent attempts to explain the origin of this emission. Cottrell and Dendy ${ }^{3}$ used a local electrostatic dispersion relation with ion Bernstein waves propagating perpendicular to the magnetic field (i.e., with parallel wave number $k_{\|}=0$ ) to suggest that the excitation is caused by the positive slope, $\partial f / \partial v_{\perp}>0$, in the velocity distribution of the protons; however, they presented no parametric comparison with experiments. Dendy, Lashmore-Davies, and $\mathrm{Kam}^{7}$ employed kinetic theory with homogeneous profiles to study the resonance of the ion Bernstein wave and the fast Alfven wave at $k_{\|}=0$ and showed that multiple cyclotron harmonics can be excited with a growth rate proportional to the square root of the proton-to-deuterium density ratio, $\left(n_{p} / n_{D}\right)^{1 / 2}$; however, they also found that the first proton harmonic is stable, which disagreas with the experimental observations. The spontaneous emission calculation of Arunasalam, Greene, and Youngs gave a spectrum of weak, broad-width harmonics. Coppi and Asherie ${ }^{9}$ calculated the growth rate, proportional to $n_{p} / n_{D}$, of the fast Alfvén instability due to $\partial f / \partial v_{\perp}>0$; however, their linear theory predicted that the first proton harmonic is stable. Coppis also studied the DT case and found that the first three alpha-particle harmonics are marginally stable. He concluded that additional processes should be considered for the excitation of the very loweat harmonics. Simultaneously, Chen ${ }^{10}$ investigated a two-energy-stream cyclotron instability caused by the weak relativistic mass effect of the fast ions in a magnetized plasma. 
The purpose of this work is to apply the general theory proposed in Ref. 10 in order to study the cyclotron emission and make detailed comparisons with experimental results in particular, the observed excitation of the first proton harmonic and the measured relative spectral amplitudes of different harmonics for both DD and DT fusion plasmas. Also, for this propose, a particle-in-cell simulation was developed in the present work, from whose results we verify the excitation mechanism of Ref. 10 and the scaling law for the saturation amplitudes.

We assume that the excited waves mainly propagate across the external magnetic field, which is valid at the rational surfaces of a tokamak, because the instability peaks where $k_{\|} \sim 0 .{ }^{10}$ When we include nonzero $k_{\|}$due to shear in the magnetic field, the electron Landau damping can be neglected for poloidal mode numbers, $m$, smaller than $q a R \omega / r v_{e}$ ( $\sim 10^{3}$ for typical tokamaks), where $q$ is the safety factor, $a$ is the minor radius, $R$ is the major radius, $\omega$ is the wave frequency, $r$ is the radial width of the wave envelope, and $v_{e}$ is the electron thermal velocity. The effect of a local nonuniform magnetic field ${ }^{11,12}$ is found to only slightly modify the instability.

When the weak relativistic mass effect is taken into account, the harmonic cyclotron frequency of the fast ions is slightly lower than the corresponding harmonic cyclotron frequency of the thermal ions. This difference gives rise to two-streaming in gyrospace. We consider frequencies $\omega$ in the range $\ell_{s} \Omega_{c s} \geq \omega \geq \ell_{f} \Omega_{c f} / \gamma$, where $\ell$ is the harmonic number, $\Omega_{c}=Z e B / m_{i} c$ is the cyclotron frequency, $Z$ is the charge number, $e$ is the unit charge, $B$ is the external magnetic field, $m_{i}$ is the ion mass, $c$ is the speed of light, $\gamma$ is the Lorentz factor of the fast ion, and the subscripts $s$ and $f$ indicate the slow and fast ions, respectively. This condition does not change if the magnetic field is nonuniform, since all the frequencies are related to the local cyclotron frequency. We will calculate the different harmonics of an electrostatic ion cyclotron wave at its peak growth for the following parameter regime: $n_{f} / n_{s} \ll 1, \omega_{p s} / \Omega_{c s} \gg 1, \omega_{p e} / \Omega_{c e} \ll 1, x,=k \rho_{s} \ll 1$, and 
$\gamma-1 \sim v^{2} / 2 c^{2} \ll 1$; here, $\omega_{p}$ is the plasma frequency, $k$ is the wave number, $\rho$ is the Larmor radius, and the subscript $e$ stands for electron. The relevant dispersion relation ${ }^{10}$ can be written as $\epsilon(\omega, k) \sim \mathcal{A}+\mathcal{D} /(\delta-\Delta)+\mathcal{C} / \delta^{2} \sim 0$, where $\mathcal{A} \sim 1$ for $\ell_{s}=1$ (e.g, the first alpha-particle harmonic, which resonates with the first deuterium harmonic), and $\mathcal{A} \sim 1-\omega_{p s}^{2} /\left[2 \Omega_{c s}\left(\ell_{f} \Omega_{c f}-\Omega_{c s}\right)\right]$ for $\ell_{s}>1 ; \mathcal{D} \sim-\left(\omega_{p s}^{2} / 2 \Omega_{s}^{2}\right)\left(x_{s}^{2} / 2\right)^{\ell_{s}-1} ; \mathcal{C} \sim\left(\omega_{p f}^{2} / k^{2} c^{2}\right)\left\langle J_{l f}^{2}\right\rangle ;$ $\delta=\omega / \ell_{f} \omega_{c f}-1$ is the normalized frequency mismatch; $\Delta=1-\gamma \ell_{s} \Omega_{c \Omega} / \ell_{f} \Omega_{c f} ; \omega_{c f}=\Omega_{c f} / \gamma_{i}$ and $\left\langle J_{\ell_{f}}^{2}\right\rangle$ is the Bessel function of the first kind of order $\ell_{f}$ averaged over a shell distribution. For $\ell_{3}>1$, this cubic dispersion re! : n yields the normalized frequency mismatch $\left.\delta \sim 0.5(\gamma-1)^{1 / 3}\left(\omega_{p f}^{2} / \omega_{p s}^{2}\right)^{1 / 3}\left(\left\langle J_{l,}^{2}\right\rangle\right)^{1 / 3} ; \quad-\Omega_{c s}^{2}\right)^{1 / 3} /(k c)^{2 / 3}$, while the growth rate is $\sqrt{3} \delta \ell_{f} \omega_{c f}$. For $\ell_{s}=1$, the growth rate, $2(\gamma-1)^{1 / 2}\left(\omega_{p f} / \omega_{p s}\right)\left(\left\langle J_{l_{f}}^{2}\right\rangle\right)^{1 / 2}\left(\ell_{f} \omega_{c f}\right)^{2} / k c$, and the normalized frequency mismatch, $\sqrt{2}\left(\omega_{p f} / \omega_{p o}\right)^{2}\left(\left\langle J_{\ell_{f}}^{2}\right\rangle\right)^{1 / 2}\left(\ell_{f} \omega_{c f} / k c\right)^{2}$, are smaller.

The instability transfers energy from the fast ions to the wave, and from the wave to the slow ions. The relative spectral amplitudes of the different harmonics are determined by the nonlinear saturation mechanisms. During the initial linear growth phase of the wave-fast ion interaction, the inequality $\omega>\ell, \Omega_{c f} / \gamma$ holds so that the fast ions are losing energy on average, and thus the relativistic factor $\gamma$ decreases on average. The interaction stops when this inequality is violated because of the $\gamma$ change, that is, when the mismatch vanishes, ${ }^{10}$ which occurs when $\langle\gamma\rangle_{\text {aturation }}=\gamma /(1+\delta)$. When we include magnetic field shear, the minimum mismatch estimated from linear theory is $\delta_{\min }=m \rho_{p} r / 2 \ell_{f} q a R ;{ }^{13}$ this is zero for a pure radial $(m=0)$ mode. $^{14}$ Define the efficiency $\eta$ in terms of the rate of change of the fast ion kinetic energy: $\eta=\left(\gamma-\langle\gamma\rangle_{\text {acturation }}\right) /(\gamma-1)$. We assume that the relative wave energy amplitudes are determined by the available fast ion energies (measured by $\eta$ ) for the different harmonics. For slow ion harmonic numbers $\ell_{0}>1$, we find $\eta \cong$ $0.5(\gamma-1)^{-2 / 3}\left(\omega_{p f}^{2} / \omega_{p s}^{2}\right)^{1 / 3}\left\langle J_{l_{f}}^{2}\right\rangle^{1 / 3}\left(\ell_{f}^{2} \omega_{c f}^{2}-\Omega_{c s}^{2}\right)^{1 / 3} /(k c)^{2 / 3}$. This is proportional to $\left(n_{f} / n_{s}\right)^{1 / 3}$. Since the total available fast ion energy is proportional to $n_{f}$, the spectral amplitude of the same harmonic for different density values scales as $\left(n_{f} / n_{0}\right)^{4 / 3}$. For $\ell_{0}=1$, we have 
$\eta \cong \sqrt{2}\left(\omega_{p f} / \omega_{p s}\right)^{2}\left(\left\langle J_{\ell_{f}}^{2}\right\rangle\right)^{1 / 2}\left(\ell_{f} \omega_{c f} / k c\right)^{2}(\gamma-1)^{-1}$, which means that the spectral peaks are proportional to $\left(n_{f} / n_{s}\right)^{2}$, and hence much smaller. We note that the effect of geometry and the processes of wave propagation and mode conversion may be involved in determining the scaling law for the wave spectral amplitudes as measured outside the tokamak plasma; however, these processes are not within the scope of the present work.

We have developed a one-space-and-three-momentum-dimensional electrostatic particlein-cell simulation of this situation, with kinetic ions and dielectric electrons. The ion dynamics can be treated relativistically or classically, which gives an important check on the importance of the mass shift mechanism. The system is periodic and is initiated with a quiet start, ${ }^{15,16}$ with particles uniformly distributed with a very small perturbation. The case to be simulated is the D-D reaction in the Joint European Tokamak (shot \#6992) ${ }^{3}$ which has parameters at the plasma edge, where the emission originates, as follows: $B=3.4 \mathrm{~T}$, Maxwellian deuterium temperature $T_{D}=1 \mathrm{keV}, n_{D}=1 \times 10^{13} \mathrm{~cm}^{-3}$, shell-distributed protons with energy $E_{p}=3.02 \mathrm{MeV}$, and $n_{p}=8 \times 10^{8} \mathrm{~cm}^{-3}$. These parameters give $\omega_{p D} / \omega_{c D}=18$, $n_{p} E_{p} / n_{D} T_{D}=0.24, \rho_{p} / \rho_{D}=55$, (where the subscript $D$ stands for deuterium), $\gamma=1.0032$, and $\rho_{p}=v_{0} / \omega_{c f} \sim 7 \mathrm{~cm}$, with $v_{0}$ the birth speed of the fusion-produced ions. In the numerical simulation, we keep the first two parameters constant to insure that the physics of interest is unchanged, and we use $\rho_{p} / \rho_{D}=3.5, \gamma=1.064$ with $5 \%$ energy spread, and $n_{p} / n_{D}=0.02$ to make the simulation affordable. The system length is 512 grids, with $d x$ the grid size (normalized to unity). The proton gyroradius is $\rho_{p}=61 d x$. Time is normalized to $\Omega_{c D}^{-1}$. One time step is 0.02 . The simulation uses 59048 deuterons and 7776 protons. The modes with mode numbers from 1 to 7 are kept; the wave number $k$ is equal to $2 \pi / 512$ times the mode number.

Figure 1 shows the time history of the field energy normalized to the total system energy. The field energy grows exponentially and saturates at $t \sim 150 \Omega_{c D}^{-1}$. In contrast, when we turn 
off the relativistic effect by using Newton's equation for the ion acceleration instead of the Lorentz equation, Fig. 1 shows that the field energy remains at about its initial noise level: i.e., this instability mechanism is removed. This comparison makes sure that the observed instability is due to the weak relativistic mass effect. To examine the effect of a nonuniform magnetic field, we added a $2 \%$ peak-to-peak sinusoidal nonuniformity over the system length and repeated the relativistic run. We observed that the linear growth rate has no appreciable change and the saturation level remains at the same level. To further clarify the instability mechanism, we calculated the growth rate for different modes from the history of their field energies. The results are shown as dots in Fig. 2, whereas the solid and dashed curves are derived from linear theory for a monoenergetic shell distribution and are for the first and second proton harmonics, respectively. This agreement, as well as the particle dynamics (not shown), verify that the growth mechanism is due to the two-energy-stream cyclotron instability.

In Fig. 3 we show the power spectrum for the two most unstable modes: mode 2 (with wavelength $\left.\lambda=256 d x=4.2 \rho_{p}\right)$ and mode $6\left(\lambda \sim 85.3 d x=1.4 \rho_{p}\right)$. The spectrum of mode 2 is dominated by the first proton harmonic, whereas the spectrum of mode 6 is dominated by the second proton harmonic. The relative amplitudes of the two spectral lines are consistent with the prediction ( $\eta=0.16$ for the first harmonic and $\eta=0.14$ for the second harmonic) irom the nonlinear saturation scaling law discussed earlier. From the scaling based on the simulation and the theory, we estimate that, for the JET deuterium-deuterium case, the first harmonic has $\eta=0.03$ at $\lambda=9 \mathrm{~cm}$ whereas the second harmonic has $\eta=0.001$ at $\lambda=1 \mathrm{~cm}$. Their relative amplitudes are consistent with the experimental results; ${ }^{3}$ unfortunately, no measurements of the wavelength are available.

For a deuterium-tritium case (shot \#26148) in $\mathrm{JET}^{5,6}$ with edge parameters $B=2.8 \mathrm{~T}$, $n_{D}=9 \times 10^{12} \mathrm{~cm}^{-3}, n_{T}=1 \times 10^{12} \mathrm{~cm}^{-3}$, and $T_{D}=T_{T}=7 \mathrm{keV}$, the proton energy is $E_{p}=3.02 \mathrm{MeV}$ and their density is $n_{p}=1 \times 10^{9} \mathrm{~cm}^{-3}$, and the alpha particles have 
energy $E_{\alpha}=3.5 \mathrm{MeV}$ and density $n_{\alpha}=8 \times 10^{9} \mathrm{~cm}^{-3}$. We expect the first alpha (deuterium) harmonic to be weak, the first proton (second alpha) harmonic with $\eta=0.08$ to peak at $\lambda=25 \mathrm{~cm}$, the second proton harmonic with $\eta=0.016$ to peak at $\lambda=4 \mathrm{~cm}$, and the third proton harmonic to peak at $\lambda=2 \mathrm{~cm}$ with $\eta=0.007$. The efficiency $\eta$ of the first proton harmonic may be lower because its wavelength is too long. Again, the spectral amplitudes are consistent with the experimental observations.

Fusion-produced tritons and helium-3 are born with energies of 1.0 and $0.82 \mathrm{MeV}$, respectively, at a rate roughly equal to that of the fusion-produced protons. At their cyclotron harmonics that are not degenerate with the deuterium harmonics, there is no spectral peak. Our explanation is that resonance of these harmonics with the deuterium harmonics (e.g., $\left.\ell_{s} \omega_{c s} \sim \ell_{f} \omega_{c f}\right)$, which is required for the instability, does not occur.

In summary, we have given an explanation for the excitation mechanism of the ion cyclotron harmonic emissions in both D-D and D-T fusion plasmas. A particle-in-cell simulation code that was developed to study the linear and nonlinear scaling laws for this collective mechanism shows that the first proton harmonic is one of the highest spectral peaks whereas the first alpha harmonic is weak. The relative amplitudes of the different harmonics were also obtained and found to be consistent with experimental observations. Without mode conversion and other effects, the spectral peak of the first proton harmonic scales with proton density as $\left(n_{p} / n_{D}\right)^{4 / 3}$, which is close to that inferred from the experiments. ${ }^{1-3}$ We note that some higher harmonics may be excited by a number of other mechanisms. ${ }^{3,6-10,14}$ However, the very lowest harmonics can only be explained by a two-energy-stream cyclotron instability from the weak relativistic mass effect. ${ }^{10}$ In order to have a full disclosure of the practical conditions for relativistic cyclotron excitation in confinement devices (tokamaks) and the resulting fast ion dynamics, more numerical and theoretical studies are needed. 
The authors wish to thank A. Gibson for providing D-T cyclotron spectral data and B. Coppi, J.M. Dawson, S. Mahajan, H. Berk, and R.D. Hazeltine for helpful discussions. This work was supported by the US Department of Energy under contract No. DE-FG05-80ET53088. 


\section{References}

${ }^{1}$ G.A. Cottrell, in Proceedings of the Course and Workshop on Applications of RF Waves to Tokamak Plasmas, Varenna, Italy, 1985, edited by S. Bernabei, U. Gasparino, and E. Sindoni (Monatypia Franchi, Perugia, 1985), Vol. 2, p. 710.

${ }^{2}$ G.A. Cottrell, P.P. Lallia, G. Sadler, and P. van Belle, in Proceedings of the 13th European Conference on Controlled Fusion and Plasma Heating, Schliersee, Germany, 1986, edited by G. Briffod and M. Kaufmann (European Physical Society, Petit-Lancy, Switzerland, 1986), Pt. 1, Vol. 2, p. 37.

${ }^{3}$ G.A. Cottrell and R.O. Dendy, Phys. Rev. Lett. 60, 33 (1988), and references therein.

${ }^{4}$ P. Schild, G.A. Cottrell, and R.O. Dendy, Nuclear Fusion 29, 834 (1989).

${ }^{5} \mathrm{JET}$ Team, Nuclear Fusion, 32, 187 (1992); also, A. Gibson, private communication (April, 1992).

${ }^{6}$ B. Coppi, Phys. Lett. A 172, 439 (1993); also, private communication (April, 1993).

${ }^{7}$ R.O. Dendy, C.N. Lashmore-Davies, and K.F. Kam, Phys. Fluids B 4, 3996 (1992); also, 5, 1937 (1993).

${ }^{8}$ V. Arunasalam, G.J. Greene, and K.M. Young, Bull. Am. Phys. Soc. 37, 1605 (1992).

${ }^{9}$ B. Coppi and N. Asherie, Bull. Am. Phys. Soc. 37, 1475 (1992).

${ }^{10}$ K.R. Chen, Bull. Am. Phys. Soc. 37, 1421 (1992), to be published in Phys. Lett. A.

${ }^{11}$ X.S. Lee, J.R. Myra, and P.J. Catto, Phys. Fluids 26, 223 (1983).

${ }^{12}$ C.N. Lashmore-Davies and R.O. Dendy, Phys. Fluids B 1, 1565 (1989). 
${ }^{13}$ A.B. Mikhailovskii, Theory of Plasma Instabilities (Consultants Bureau: New York, 1974), Vol. 2, p. 151.

${ }^{14}$ T.D. Kaladze and A.B. Mikhailovskii, Sov. J. Plasma Phys. 1, 128 (1975).

${ }^{15}$ J.M. Dawson, Rev. Mod. Phys. 55, 403 (1983).

${ }^{16}$ C.K. Birdsall and A.B. Langdon, Plasma Physics via Computer Simulation (Adam Hilger: New York, 1991), p. 394. 


\section{Figure Captions}

1. Time history of the field energy for the relativistic and nonrelativistic cases.

2. Growth rate for the first and second proton harmonics vs. wavelength, from simulation (dots) and theory (curves).

3. The power spectrum for the two most unstable modes (mode 2 and mode 6). 


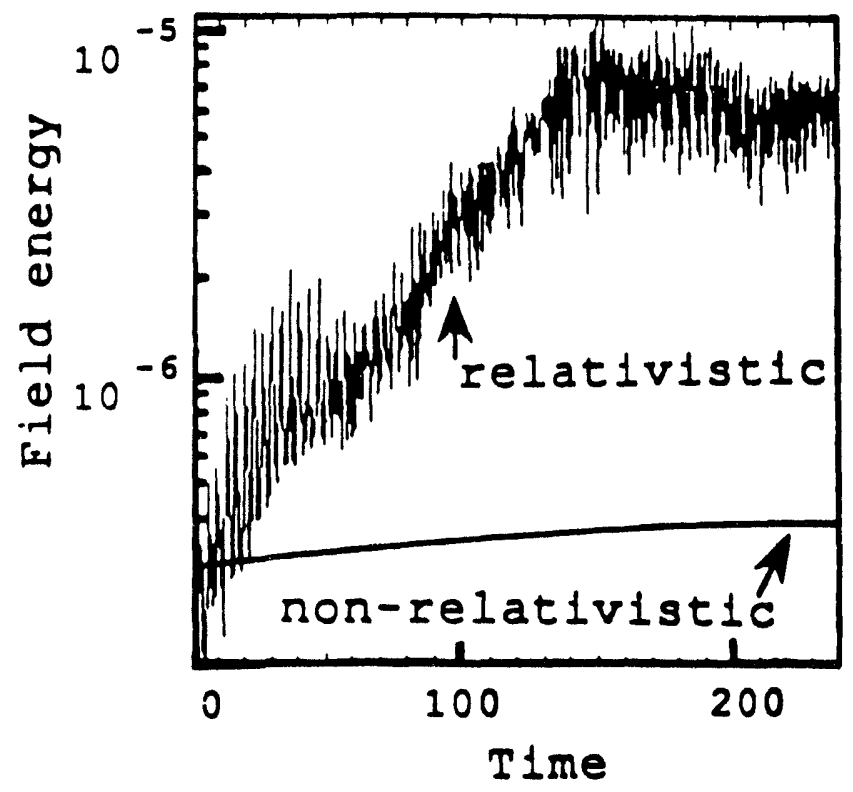

Fig. 1. Chen et al. 


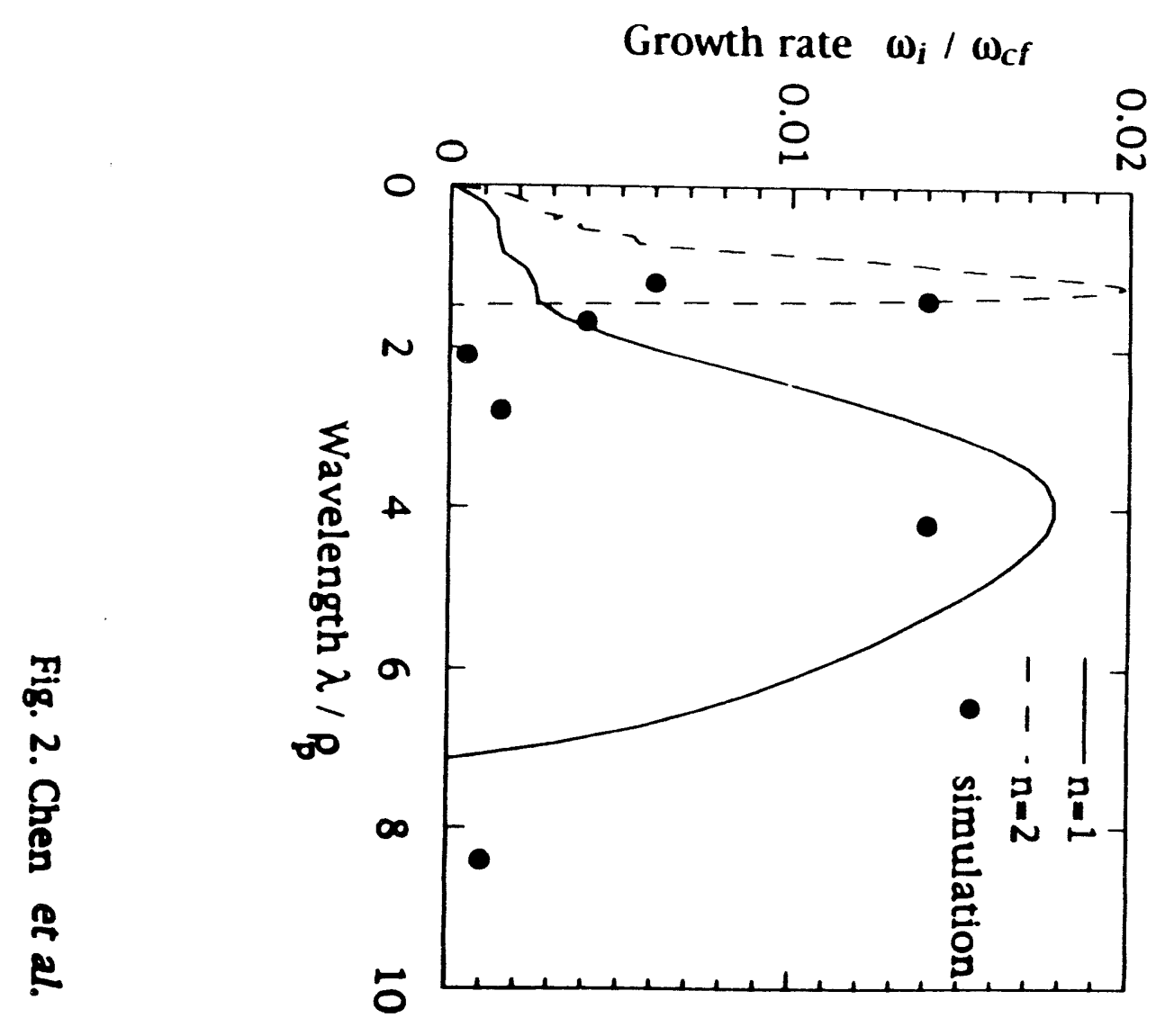




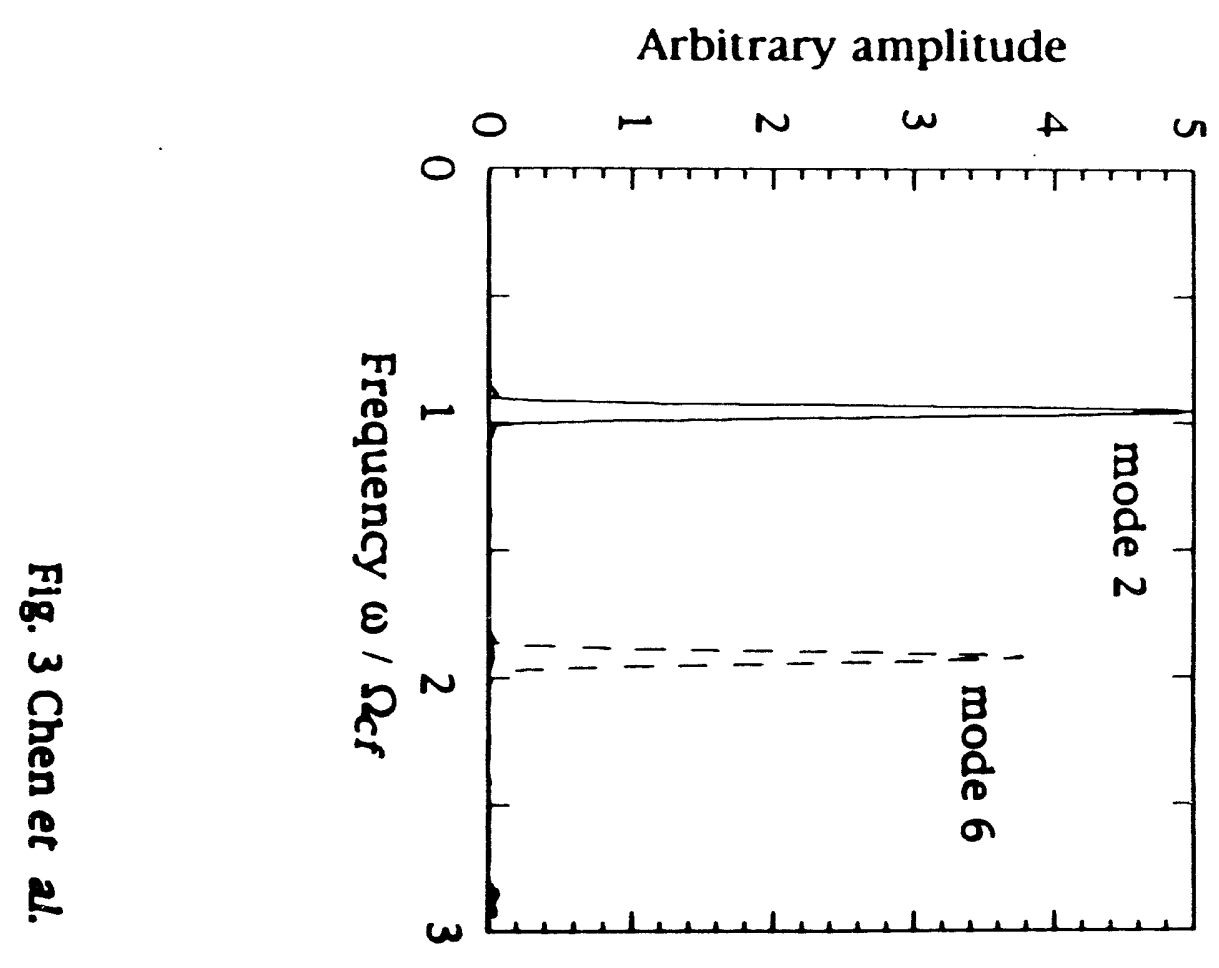



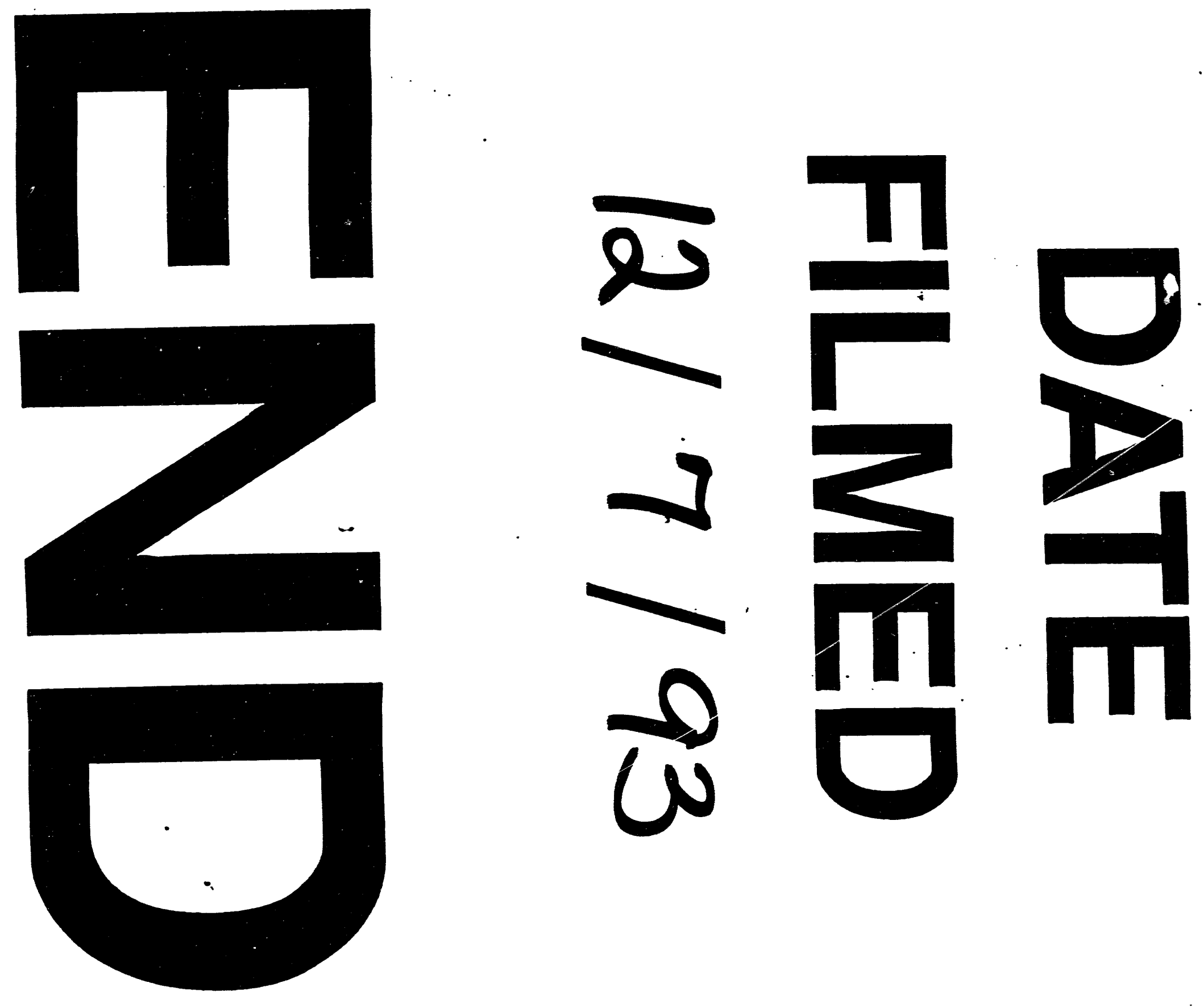
